\title{
Multiwavelength Photometry of the Young Intermediate Mass Eclipsing Binary TY CrA
}

\author{
M. Ammler-von Eiff ${ }^{1}$, M. Van̆ko ${ }^{2}$, T. Pribulla ${ }^{2}$, E. Covino ${ }^{3}$, \\ R. Neuhäuser ${ }^{4}$, and V. Joergens ${ }^{5,6}$ \\ ${ }^{1}$ Thüringer Landessternwarte Tautenburg, Sternwarte 5, 07778 Tautenburg, Germany \\ email: ammler@tls-tautenburg.de \\ ${ }^{2}$ Astronomical Institute of the Slovak Academy of Sciences, 05960 Tatranská Lomnica, \\ Slovakia \\ email: vanko@ta3.sk, pribulla@ta3.sk \\ ${ }^{3}$ INAF - Osservatorio Astronomico di Capodimonte, Salita Moiariello, 1680131 - Napoli, Italy \\ ${ }^{4}$ Astrophysikalisches Institut und Universitäts-Sternwarte, Schillergäßchen 2-3, 07745 Jena, \\ Germany \\ ${ }^{5}$ Max-Planck-Institut für Astronomie, Königstuhl 17, 69117 Heidelberg, Germany \\ ${ }^{6}$ Zentrum für Astronomie Heidelberg, Institut für Theoretische Astrophysik \\ Albert-Ueberle-Str. 2, 69120 Heidelberg, Germany
}

\begin{abstract}
One of the handful of known PMS eclipsing binaries is a component of the spectroscopic triple TY CrA. Its secondary component is particularly interesting since it is a star of relatively high mass $\left(1.64 M_{\odot}\right)$ which is still on the pre-main sequence. The eclipsing binary was analyzed in the optical wavelength range $\sim 10$ years ago, however, the crucial secondary eclipse minimum is very shallow. Therefore, we are obtaining new photometry in both optical and near-IR bands. We present first observations in (BVRI) which show that the secondary eclipse depth increases to about $0.1 \mathrm{mag}$ in the $I$ band. The increased eclipse depth with respect to other bands will help to better determine the colours and dimensions of the system. Furthermore, we show and discuss first near-IR observations of the primary eclipse. In addition to the light curves we are obtaining radial velocities in order to pin down the orbital parameters of the triple. Our first observations agree with the orbital parameters derived $\sim 10$ years ago.
\end{abstract}

Keywords. binaries: eclipsing, stars: fundamental parameters, stars: individual (TY CrA) etc.

\section{Introduction}

TY CrA is a hierarchical triple, maybe even quadruple system, embedded in a reflection nebula in the CrA star forming region. Two components form a massive eclipsing doublelined spectroscopic binary with an orbital period of almost 3 days. A third spectroscopic component is in a wide orbit around the eclipsing pair (Casey et al. 1995; Corporon et al. 1996). A visual fourth component was detected by Chauvin et al. (2003).

\section{Overview}

The eclipsing binary has been analysed in detail previously but uncertainties remain. Casey et al. (1998) obtained optical light curves of the eclipsing binary in uvby. They derived component masses of $3.16 \pm 0.02 M_{\odot}$ and $1.64 \pm 0.01 M_{\odot}$. The latter is particularly interesting since it has not yet reached the main sequence. Its properties are uncertain since the light curve analysis is challenging.

The secondary minimum is very shallow and the out-of-eclipse variability is larger than the measurement errors (Vaz et al. 1998). A few years ago, the benefits of observations 

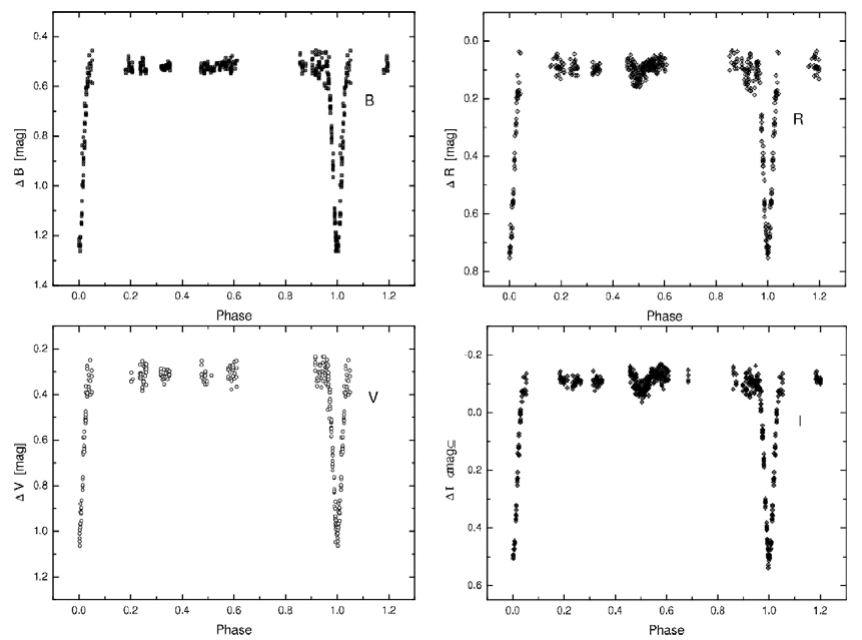

Figure 1. BVRI light curves obtained with VYSOS6 at Cerro Armazones, Chile.

in the near-infrared (NIR) have been demonstrated. Covino et al. (2004) analysed an eclipsing binary in the optical and NIR and noticed that the depth of the secondary minimum is more pronounced in the NIR. The error bars on the stellar parameters could be reduced by $\approx 80 \%$ compared to previous analysis without NIR data (Covino et al. 2000). The goal of the present work is to analyse TY CrA in a way similar to Covino et al. (2004) and to get more precise secondary parameters by observations in the NIR. New photometric observations of TY CrA have been obtained by the authors in recent years in both optical and NIR bands. The primary minimum was observed in 2006 with SOFI at La Silla, Chile. New optical data and additional NIR photometry have been obtained from 2009-2011 with VYSOS6 at Cerro Armazones, with REM at La Silla, and ANDICAM at Cerro Tololo, Chile. First results have been obtained. The minimum time derived from the SOFI light curves differs by 7 minutes from the ephemeris presented by Casey et al. (1998) which is indicative of additional components. We reproduced the stellar and orbital parameters derived by Casey et al. (1998) based on the 2009 VYSOS6 data (Fig. 1) using the methods of Pribulla et al. (2008). It can be noticed that the secondary minimum at a phase of $\approx 0.5$ already becomes more pronounced in the $R$ and $I$ bands, compared to the $B$ and $V$ bands.

\section{References}

Casey, B. W., Mathieu, R. D., Vaz, L. P. R., Andersen, J., \& Suntzeff, N. B. 1998, AJ, 115, 1617

Casey, B. W., Mathieu, R. D., Suntzeff, N. B., \& Walter, F. M. 1995, AJ, 109, 2156

Chauvin, G., Lagrange, A., Beust, H., Fusco, T., Mouillet, D., Lacombe, F.,Pujet, P., Rousset, G., Gendron, E., Conan, J. M., Bauduin, D., Rouan, D., Brandner, W., Lenzen, R., Hubin, N., \& Hartung, M. 2003, A\&SA, 406, L51

Corporon, P., Lagrange, A. M., \& Beust, H. 1996, A\&A, 310, 228

Covino, E., Catalano, S., Frasca, A., Marilli, E., Fernández, M., Alcalá, J. M., Melo, C., Paladino, R., Sterzik, M. F., \& Stelzer, B. 2000, $A \& A$, 361, L49

Covino, E., Frasca, A., Alcalá, J. M., Paladino, R., \& Sterzik, M. F. 2004, A\& A, 427, 637

Pribulla, T., Baludanský, D., Dubovský, P., Kudzej, I., Parimucha, Š., Siwak, M., \& Vaňko, M. 2008, MNRAS, 390, 798

Vaz, L. P. R., Andersen, J., Casey, B. W., Clausen, J. V., Mathieu, R. D., \& Heyer, I. 1998, $A \mathscr{E} A S, 130,245$ 